\title{
Terrorisme et politiques de dépossession(s) dans Le serment des barbares de Boualem Sansal
}

Karim Zakaria Nini

Université de Montréal

Dans l'analyse qui va suivre, nous proposons d'étudier un cas de dépossession contemporain et assez particulier que nous offre l'écrivain algérien de langue française, Boualem Sansal. Né en Algérie dans un petit village de la wilaya de Tissemssilt, Sansal fait des études à l'École polytechnique d'Alger et obtient un doctorat en économie. Haut fonctionnaire de l'État au ministère de l'Industrie, l'écrivain finit par être licencié à cause de ses écrits très critiques à l'encontre du système politique algérien; des écrits qui se sont enchaîné après la parution de son premier roman Le serment des barbares, qui sera au cœur de notre l'étude.

Publié en 1999, ce roman nous immerge dans l'Algérie de la fin des années 1990. Ce roman policier à l'atmosphère lourde nous plonge dans les rues lugubres de la ville de Rouiba, une ville décrite comme paisible et accueillante avant d'être ravagée par des décennies de politiques d'industrialisation anarchique et par un phénomène de terrorisme chronique. Nous verrons dans notre analyse comment une succession de politiques autoritaires a fini par déposséder la ville de Rouiba de sa nature de petite ville agricole, mais aussi comment le terrorisme et la guerre durant la «Décennie noire » ont déstructuré la société en modifiant de façon violente le mode de vie de citoyens totalement enterrés sous le poids d'évènements qui les submergent. Cette dépossession comme nous allons le voir s'opère sur toute la société, c'est un phénomène de masse qui va agir comme une maladie et un dysfonctionnement au niveau des relations sociales, car dans un environnement de guerre, l'autre est la mort.

\section{1. «Dispossession"}

Dans Dispossession: The Performative in the Political, Athanasiou et Butler explorent le concept de la performativité avec en toile de fond une réflexion éclectique sur les différentes formes de politiques d'oppression qui caractérisent l'ère néolibérale. C'est ainsi qu'est introduit le concept de Dispossession, que nous allons traduire en « dépossession » et qui théorise les différentes violences que connaît l'individu soumis malgré lui à des politiques qui le dépassent et sur lesquelles il a de moins en moins d'influence. Les deux auteures expliquent que la dépossession est une violence que vit l'individu exposé à différents types de politiques dures allant de la colonisation et l'esclavage à l'assujettissement néolibéral :

Being dispossessed refers to processes and ideologies by which persons are disowned and abjected by normative and normalizing powers that define cultural 
intelligibility and that regulate the distribution of vulnerability : loss of land and community, ownership of one's living body by another person, as in histories of slavery, subjection to military, imperial and economic violence, poverty, securitarian regimes, biopolitical subjectivation, liberal possessive individualism, neoliberal governmentality, and precarization. (Butler, Athanasiou 9)

Ainsi, le concept de dépossession théorise un ensemble assez conséquent de phénomènes par lesquels un individu subit une violence dans sa manière de vivre dont il n'est plus maittre, il est ainsi vulnérable face à une force supérieure et perd son autonomie et son pouvoir de décision. Ces phénomènes qui sont le plus souvent des politiques économiques et militaires créent chez l'individu une vulnérabilité totale à des politiques sur lesquelles il n'a aucune emprise. Il est ainsi condamné à les subir et à souffrir de leurs conséquences qui peuvent aller de la perte de la terre et du mode de vie dans des cas tels que la colonisation à la perte du pouvoir de décision au niveau économique. On parlera par exemple de politiques d'endettements qui, si elles ne sont pas rigoureusement maitrisées conduisent souvent l'individu à l'hypothèque de ses biens ou, dans cas plus extrêmes même s'ils sont rares, au suicide. La vulnérabilité totale de l'individu à un pouvoir qui le domine et qui le dépossède de son indépendance assujettit ce dernier, il perd donc son individualité.

Cet assujettissement est justement expliqué dans le deuxième sens que prend le concept de dépossession qui se veut une théorisation exhaustive des phénomènes par lesquels l'individu subit une perte infligée par un pouvoir oppressant. L'assujettissement qui peut être associé à une perte de soi et de son individualité est le résultat premier de la dépossession découlant de pouvoirs normalisant qui dépossèdent l'individu de son libre arbitre le poussant ainsi à suivre un courant de pensée ou à vivre un mode de vie «par défaut ». L'individu est entraîné sans qu'il ne s'en rende compte dans un mouvement de masse se retrouvant par exemple à faire la même chose que d'autres membres de sa communauté, car la majorité le fait. Nous parlerons par exemple des traditions auxquelles se soumettent certaines sociétés et qui ne sont que la répétition de rituels symboliques hérités par d'anciennes générations; ces traditions si elles servent de ciment dans le fonctionnement d'une communauté humaine en structurant et raffermissant la cohésion sociale peuvent constituer un facteur important de dépossession dans certains cas en agissant comme une force oppressante dépossédant l'individu de son pouvoir de décision. On parlera du voile dans certaines sociétés musulmanes, qui est en l'espace de quelques années devenu un phénomène social auquel la femme doit se soumettre afin d'être acceptée par la communauté. Nous donnerons aussi l'exemple du mariage qui dans beaucoup de cultures sert encore des intérêts économiques ou politiques au détriment des choix personnels des concernés :

[...] dispossession encompasses the constituded, preemtive losses that condition one's being dispossessed (or letting oneself become dispossessed) by another: 
one is moved to the other and by the other - exposed and affected by the other's vulnerability. The subject comes to « exist » by installing within itself lost objects along with the social norms that regulate the subject's disposition to the address of the other. (9)

La nature du rapport à l'autre constitue un élément important dans le processus de dépossession, c'est en effet dans la nature de la relation qu'on entretient avec «l'autre » que s'explique la majorité de nos choix de vie, l'on décidera d'acheter telle marque de vêtements, car la majorité de nos amis s'habillent avec, ou on décidera d'éviter tel métier, car l'on entretient une relation conflictuelle avec le modèle que l'on a eu de son père. Dans les deux exemples nous pouvons remarquer que les choix que l'on prend, et qui peuvent varier entre de banales décisions à d'autres qui définissent le cours d'une vie, nous sont dictés dans la plupart des cas par la nature de la relation que l'on entretient avec des personnes de notre entourage. La question du libre arbitre se pose ici; un libre arbitre duquel on est dépossédé à partir du moment où notre existence dépend de notre intégration à un mouvement de masse qui nous entraîne, ou encore d'une relation conflictuelle avec un « autre».

Butler et Athanasiou décrivent l'individu comme un être éminemment social rappelant ainsi les limites de ce qu'elles appellent «l'autonomie du sujet». Ce dernier est confronté à l'altérité et à sa dépendance vis-à-vis des autres membres de sa communauté, cette vulnérabilité comme nous allons le voir, alliée à des phénomènes violents tels que le terrorisme, va radicalement modifier le mode de vie de la société jusque dans ses fondements les plus élémentaires. Si, par ailleurs, le concept de dépossession développé dans l'ouvrage de Butler et Athanasiou peut parfaitement s'expliquer dans le cas de la ville de Rouiba telle que présentée dans l'incipit du Serment des barbares, la théorisation du concept contient tout de même un détail sur lequel il est utile de se pencher afin de développer notre problématique. En parlant des limites de l'autonomie du sujet néolibéral, les deux auteurs nous mettent face à un cas spécifique de dépossession qui ne s'applique pas à la situation algérienne à l'époque où se déroulent le récit dans premier roman de Sansal.

Chez Sansal, la société est confrontée à la fois à un phénomène d'industrialisation socialiste qui va s'avérer être un désastre urbain pour la ville de Rouiba, mais aussi à une réalité de plus en plus dynamique en ce début de XXIe siècle: le terrorisme. Notre problématique est donc la suivante : comment s'opère dans le texte de Sansal la double dépossession de l'individu et son assujettissement face aux deux phénomènes que sont le terrorisme et l'industrialisation menée superficiellement? La question étant formulée, il est nécessaire d'expliquer ce que nous entendons par « industrialisation menée superficiellement», car si l'Europe a connu une industrialisation certes chaotique durant ces premières années, mais qui 
s'est bâtie sur des décennies d'ébullition intellectuelle et sociale, rien ne prédisposait l'Algérie nouvellement indépendante à aller vers l'industrialisation si ce n'est «la prise de décision politique ». Le pays qui sortait de plus de 130 années de colonisation faisait face à de nombreux défis parmi lesquels la modernisation des structures sociales ainsi que l'alphabétisation d'une partie importante de la population rurale qui n'a connu pendant plus d'un siècle que l'extrême précarité. La modernisation du tissu économique et la décision de mettre le pays sur les rails de l'industrialisation nécessitaient donc beaucoup plus qu'une simple volonté politique :

De nos jours, trente années après l'indépendance, elle est regardée comme le fleuron de l'industrialisation du pays. Par décret, elle a été classée "ville industrielle », et offerte à l'administration de cohortes moutonnières de visiteurs d'usine que des guides frénétiques et arrogants mènent à la baguette. (Sansal 9)

La façon dont Sansal dépeint le quotidien de la ville de Rouiba dans sa fiction nous permet d'avoir une idée sur la situation socio-économique de l'Algérie des années quatre-vingt-dix. Dans cet extrait, il nous est permis d'appréhender l'absurdité de cette classification d'une région à tradition agraire en ville industrielle, par décret. Le fait que cette transformation ait été "décrétée » nous ramène au concept de dépossession tel qu'il est développé par Butler et Athanasiou: "Yet dispossession is precisely what happens when populations lose their land, their citizenship, their means of livelihood, and become subject to military and legal violence. » (10). Le décret, qui ne passe par aucune voie démocratique, est une décision politique prise et imposée par le haut, et dont le citoyen observe impuissant les retombées. Son caractère violent propulse toute une région dans l'industrialisation en omettant le caractère agricole ancestral de cette dernière. Il aura de lourdes répercussions sur le citoyen dont le mode de vie sera brusquement affecté. Décréter la ville de Rouiba ville industrielle est une action qui s'apparente à de la violence légale dont les répercussions sont certaines sur les populations non préparées à un tel basculement. Nous proposons donc dans ce travail d'analyser les effets de cette dépossession due à des politiques autoritaires tels qu'ils sont représentés dans l'incipit du premier roman de Sansal Le serment des barbares.

\section{Terrorisme et dérèglement social}

Dans notre analyse, nous nous intéresserons d'abord au terrorisme et à ses effets sur la vie des citoyens, nous verrons comment ce phénomène se répercute sur le mode de vie de la société et sa culture. L'objectif de notre analyse sera de montrer comment se manifeste dans la fiction de Sansal la réaction des membres d'une communauté face à une longue exposition au terrorisme, car si le phénomène est maintenant connu des pays occidentaux notamment depuis le 11 septembre 2001, aucun pays occidental n'a, à ce jour, été exposé près de dix ans durant et au quotidien, à la menace terroriste. Nous verrons ainsi comment la dépossession s'opère au niveau 
des citoyens de la ville de Rouiba telle que celle-ci nous est décrite par Sansal dans son roman. Le serment des barbares s'ouvre ainsi sur cette citation qui nous introduit sans ménagement dans le quotidien de la Décennie noire tel qu'il est dépeint par l'auteur :

Le cimetière n’a plus cette sérénité qui savait recevoir le respect, apaiser les douleurs, exhorter à une vie meilleure. Il est une plaie béante, un charivari irrémédiable; on excave à la pelle mécanique, on enfourne à la chaîne, on s'agglutine à perte de vue. Les hommes meurent comme des mouches, la terre les gobe, rien n'a de sens. (Sansal 7)

Il est intéressant de remarquer que le roman s'ouvre sur le mot «cimetière ». Ce mot donne le ton de l'atmosphère qui va caractériser la description des lieux dans cet incipit où la mort est omniprésente, plus que la vie. La personnification du cimetière qui «n'a plus» sa sérénité nous renvoie à la perte: le cimetière avait, et il n'a plus. Si «avoir» est "posséder", il s'agit alors bien de dépossession dans ce lieu hautement symbolique de la culture d'un groupement humain. En archéologie, l'étude des sépultures offre des informations précieuses sur «les morts $[. .$.$] et à$ travers eux sur la société des vivants et sa structure socio-économique » (Duday, Courtaud, Crubezy, Sellier, Tillier 30). La façon dont les hommes enterrent leurs morts nous renseigne sur le respect qu'ils accordent à la vie, dans le passage tiré du roman de Sansal, le cimetière est décrit comme une "plaie béante », une blessure dans la terre que les pelles mécaniques ne cessent d'élargir et dans laquelle s'amassent les morts. La scène est violente, la mise en terre des morts est décrite d'une manière crue, pas de rituels ni de respect pour ce moment symbolique censé être un moment de haute spiritualité et de recueillement, la terre est creusée à l'aide de pelles mécaniques qui « enfournent » les morts «à la chaîne ».

Quand un groupement humain ne sait plus enterrer ses morts, c'est la culture de cette communauté avec ses fondements les plus élémentaires qui s'effondre. Quand on n'a pas de respect pour la mort, c'est la vie qui n'a plus de valeur. Le terrorisme est donc un phénomène qui ne dépossède pas seulement les citoyens de leur individualité les assujettissant à une terreur qui, comme nous allons le voir, les pousse à faire corps avec la masse, mais à une échelle plusieurs fois plus grande. Il dépossède toute une société de ses structures culturelles les plus basiques remettant ainsi en cause l'existence même de cette communauté. Les rituels servent en effet de ciment au sein d'une communauté. Participer à un rituel, c'est consolider son appartenance à un groupe humain déterminé. Selon Goffman (102), les rituels participent à la socialisation et structurent le groupe. L'existence de rituels renseigne donc sur le bon fonctionnement d'une société, la disparition de ces rituels signifierait donc le contraire. Le fait que le cimetière ait perdu sa sérénité est symptomatique d'un mal qui sévit, comme un cancer, au plus profond des structures sociales d'une communauté. 
Le processus de dépossession qu'enclenche le terrorisme au niveau de l'individu cette fois est lui aussi complexe et fascinant à comprendre. Grâce au premier roman de Sansal, nous pouvons avoir un aperçu sur la nature des relations qu'entretiennent les citoyens de la ville de Rouiba soumise au terrorisme, notamment à travers le passage suivant : «La foule, qui assistait à la mise en terre de $\mathrm{Si}$ Moh, bruissait comme un essaim d'abeilles dérangé dans ses coutumes. La rumeur marchait à tire-d'aile. À la dernière pelletée, tout a été dit sur la mort de Moh. » (Sansal 21)

La scène décrit l'enterrement d'un notable de la ville de Rouiba, riche commerçant assassiné par des terroristes. Beaucoup de personnes assistent à l'enterrement de celui qu'on nomme «Si Moh», connu de toute la petite ville. Il est intéressant d'analyser la description de la scène par l'auteur qui présente la nombreuse assistance comme une "foule» et comme un «essaim d'abeilles». Le mot «foule» est un terme qui désindividualise, car on ne pense pas chaque membre individuellement, mais le groupe en entier, les membres qui composent cette foule ne sont pas importants dans leur individualité, mais seulement dans leur appartenance à cette foule qu'ils composent. L'auteur confirme ensuite cette impression en décrivant l'assistance comme un "essaim d'abeilles », les abeilles sont des insectes connus pour leur sens de la hiérarchie, les ouvrières dans le cas qui nous intéresse représentent une caste à l'intérieur de la ruche dont le but est de travailler pour l'intérêt de la colonie, on parlera d'ouvrières et non «d'une » ouvrière : l'abeille ouvrière se fond dans la masse des autres ouvrières dans l'accomplissement des tâches nécessaires à la survie de la colonie.

Cette comparaison avec un essaim d'abeilles par l'auteur accentue l'impression de désindividualisation, le membre de l'assistance perd son individualité en se fondant dans la masse de la foule, il ne fait plus qu'un avec cette dernière, il n'existe plus en tant qu'individu, mais en tant que membre du groupe. Il n'est pas ensuite anodin que l'auteur parle de «rumeur» dans cette citation, c'est un détail qui est d'une importance capitale dans la compréhension des rapports qu'entretiennent les membres de la société décrite. Les personnes qui assistent à l'enterrement de Si Moh parlent entre eux des circonstances de la mort de ce dernier, la rumeur «bruissait» pour utiliser les termes de l'auteur ce qui nous donne une impression de malaise par rapport à une bienséance qui n'a pas été respectée. L'enterrement qui est censé être un moment de recueillement et d'ultime respect pour la vie du défunt se transforme en lieu où on fait et refait le décès en alimentant la rumeur et les dires. La vie quotidienne de la société de la petite ville de Rouiba est ainsi rythmée par ces morts qui se succèdent, et des histoires qu'on pourra se raconter après comme le décrit la citation suivante: " Racontars et belles versions marchaient vite. Tantôt, dans l'intimité embaumée des cafés maures, ils se rejoindront pour se 
féconder dans l'anarchie et accoucher du jamais-vu sur la planète; jusqu'à la prochaine victime; jusqu'au prochain scandale. » (25)

Les discussions entre les personnes d'une même communauté peuvent être un corpus riche en informations sur la nature des relations qu'entretiennent entre eux les membres de la société. Dans le cas de la ville de Rouiba, confrontée à la mort au quotidien, les discussions prennent une nature macabre et malsaine. On ne parle que de morts et de scandales, les rumeurs sur la mort de telle ou telle personne courent les rues de la ville participant ainsi à la perte de la sérénité et de l'apaisement nécessaires au deuil. Ce besoin de parler à l'infini des morts et des circonstances de leur trépas nous renseigne sur l'interdépendance des membres de la communauté. Dans la scène du cimetière, ils formaient une foule, un essaim qui bruissait, car les rumeurs commençaient déjà à fleurir. La façon avec laquelle on insiste dans le texte sur ces «racontars» informe sur l'ampleur qu'a pris ce phénomène, les citoyens de la ville de Rouiba, exposés au terrorisme et à la mort au quotidien se sont formés en " foule » afin de faire face au choc, l'individu s'efface au profit du groupe. Nous assistons là à une réaction d'autodéfense d'une communauté face à un choc externe, une réaction particulière où les membres de la société ressentent le besoin de parler à l'infini du malheur qui frappe leur cité comme dans une espèce de thérapie collective à grande échelle. Butler et Athanasiou insistent sur la réduction de l'autonomie du sujet comme conséquence de la dépossession, elles affirment que «[...] dispossession can be a term that marks the limits of self-sufficiency and that establishes us as relational and interdepenant beings. » (10).

Le concept de dépossession tel que développé dans l'ouvrage de Butler et d'Athanasiou nous apprend que l'individu est dépossédé dans sa relation avec l'autre. Dans le cas de la société de la ville de Rouiba telle que présentée dans le roman de Sansal, ce qui est confirmé par l'interdépendance des membres de la communauté afin de faire face à une violence qui leur est imposée. Nous dirons dans ce cas que cette dépossession est la seule voie possible pour les citoyens de la petite ville afin de faire face au non-sens de toutes ces morts. Les rumeurs et les racontars qui peuvent apparaître comme des phénomènes malsains sont des tentatives de donner un sens à toutes ces morts qui s'enchaînent, seule façon de supporter psychologiquement le choc. Faire face au malheur en groupe est plus facile que d'y faire face seul, le citoyen inconsciemment ou non choisit d'être dépossédé de son existence en tant qu'individu autonome. Une fois Si Moh enterré, Sansal conclut par ce passage : «Les morts en terre, la foule des vivants évacue le cimetière; à pas rétifs, la tête lourde, le cœur gros, le regard vitreux, la pensée paradoxale; “c'est en ville, parmi les vivants, que la mort frappe", se disent-ils, perturbés, en cherchant ce que pourrait être le pendant de cette anomalie. » (Sansal 31) 
Ce dernier passage clôt la scène de l'enterrement de Si Moh et présente une dichotomie symptomatique du grave dérèglement qui frappe les structures sociales de la ville de Rouiba telle qu'elle nous est exposée dans le premier roman de Sansal. L'auteur parle en effet de morts et de vivants, cette dichotomie morts/vivants est une manifestation évidente de la dépossession qui s'opère sur les membres de la société de la ville soumise au terrorisme. Dans la ville de Rouiba l'on n'est plus médecin, enseignant, policier ou musicien, l'on est plus homme, femme et enfant, le terrorisme qui fait s'abattre la mort de façon aveugle a transformé la société en citoyens morts et en citoyens vivants. Il est important de constater que le mot «mort» est au pluriel, nous parlons « des» morts qui encore une fois sont pensés dans leur pluralité, dans le groupe qu'ils forment, distinct de celui des vivants. La mort d'une personne est systématiquement appréhendée dans sa relation aux autres morts, elle n'est pas importante en soi, la personne décédée ira rejoindre les autres personnes mortes avant elle.

\section{Industrialisation et colonisation: des substrats de dépossessions}

La double dépossession qui touche les citoyens de la ville de Rouiba est aussi causée par une succession de politiques dévastatrices pour le mode de vie des citoyens de cette petite région à vocation agricole. Nous avons en effet abordé dans notre problématique une industrialisation décidée de façon bureaucratique et qui allait s'avérer sur le terrain un véritable drame pour l'urbanisme de la ville. Ce basculement « décrété » de ville agricole à ville industrielle agit indéniablement comme un facteur de dépossession étant donné les répercussions d'un tel changement sur le mode de vie des habitants de la ville de Rouiba. Un changement qui est présenté très clairement par l'auteur comme étant négatif comme en atteste ce passage : "On l'a voulue impressionnante, digne du génie du Dictateur. Elle est fébrile, morne et inquiétante, comme une cité qui se prépare à la guerre. Elle est une verrue cancéreuse sur le flanc oriental de la capitale. » (9)

La description identifie clairement le changement qui a eu lieu à Rouiba comme un échec sur le plan urbain. La ville de Rouiba est non seulement défigurée, mais elle est devenue un danger pour la capitale. L'auteur la comparant à un cancer, nous pouvons déduire que le mal qui sévit à Rouiba risque de se propager aux villes environnantes. C'est d'ailleurs le cas dans ce passage où la ville est comparée à un prédateur insatiable :

Avec le temps, la ville s'est abâtardie, boursouflée, crevassée, déformée et n'a pas fini de s'égarer. Elle s'est attifée d'acier rébarbatif et de béton scrofuleux qui lui donnent des airs revêches. [...] La ville explosa dans un tonnerre de poussière. Rageusement, elle avala les jolies fermettes qui l'environnaient, puis se rua sur les douars qui creusaient leur trou loin des regards. (13) 
Nous pouvons dans ce long passage apercevoir les effets de la politique d'industrialisation sur Rouiba, l'enchaînement d'adjectifs à connotation négative a pour effet d'accentuer l'impression que la ville a été défigurée par l'industrie. Les expressions utilisées sont par ailleurs loin d'être anodines, nous pouvons relever l'utilisation des mots « acier », « béton » ou encore «explosion» qui rappellent l'industrie lourde, et nous donne un sentiment d'une lourdeur qui s'est abattue sur la ville comme un boulet de canon. L'auteur donne vie ensuite à la ville lorsqu'il explique comment elle a «avalé » les fermes qui se trouvaient dans son environnement immédiat. Notons que les «fermettes» sont décrites à l'aide d'une caractérisation positive ce qui contraste avec la série d'adjectifs négatifs à l'aide desquels est décrite Rouiba (boursouflée, crevassée, déformée). Le fait que les petites fermes qui se trouvent autour de la ville de Rouiba soient décrites comme étant «jolies» fait ressortir une opposition que l'on peut formuler dans la dichotomie suivante: laid/joli. La ville de Rouiba, crevassée et enlaidie à cause de l'industrialisation, tel un cancer, se rue sur les petites et jolies fermes et les petits villages (douars) qui l'entouraient.

Ce contraste que fait ressortir l'auteur met l'accent sur la faillite de la politique d'industrialisation qui a radicalement modifié le visage de cette petite ville agricole. Une politique qui a non seulement modifié le mode des vies des habitants, mais qui a en plus dépossédé les habitants de leurs biens matériels, de leurs terres et de leurs propriétés. L'auteur présente l'industrialisation comme une force de colonisation dans la mesure où elle dépossède les gens de leur mode de vie et de leurs terres. Dans la citation précédente, Sansal présente la ville de Rouiba comme une force insatiable qui « avale » les jolies fermettes et les douars (petits villages) qui l'entourent. La ville s'appropriant ses lieux, leurs propriétaires originels en sont dépossédés dans le sens de "disowned ». La perte de la propriété rappelle en Algérie des évènements douloureux étant donnée l'histoire du pays qui est passée par plus de 130 ans de colonisation. Nous pouvons mieux appréhender la comparaison que fait l'auteur dans la citation suivante :

Mais rien n'arriva de plus que ce qui était déjà parti. Les complexes industriels, tels des chars d'assaut, ont envahi les domaines des colons; les ateliers privés, rémoras des grands combinats publics, se sont infiltrés dans les chais des viticulteurs et les caves des distillateurs; et, dans la foulée du déclin programmé de l'agriculture, les halles aux fruits et légumes qui pullulaient autour de la ville et embaumaient sa tiède et suave atmosphère furent investies et transformées en entrepôts d'État. Des bradages métalliques et des dispositifs tatillons sont venus les protéger de toute atteinte (11)

L'auteur décrit les complexes industriels comme des envahisseurs qui prennent possession des «domaines des colons». Nous devons nous arrêter à ce niveau de la citation afin d'essayer d'expliquer la présence du mot «colon » étant donné que le contexte du récit se déroule dans l'Algérie indépendante. Pour expliquer ce que l'auteur veut dire par «les domaines des colons ", il faut aller chercher dans l'imaginaire de l'Algérien qui a vécu 
les deux périodes de la colonisation et de l'indépendance. De façon très simpliste, le plus élevé des échelons sociaux pour l'Algérien colonisé était de vivre la vie du colon, d'avoir ce qu'il possède. Dans cette dichotomie sociale qu'impose la colonisation, la hiérarchie est claire : ou on est colon et on bénéficie de tous les privilèges que peut offrir le pays, ou on est colonisé et on se bat pour la survie avec peut-être le rêve de vivre un jour la vie du colon. Au lendemain l'indépendance du pays, les Algériens se sont rués sur les biens que les colons ont abandonnés, dans l'imaginaire collectif donc, ces biens sont ceux qu'on a pris aux colons. Même l'indépendance recouvrée, l'on ne peut séparer le colon des biens acquis, les stigmates de la colonisation sont tellement profonds que quarante-ans après l'indépendance, on continue à lier les domaines à leurs anciens propriétaires. Nous pouvons ici apercevoir une troisième dépossession qui est celle que la colonisation laisse dans les esprits et dans l'imaginaire collectif. Même appartenant désormais aux Algériens, les domaines restent ceux des colons dans les esprits et les mémoires.

Il ressort ensuite au regard de l'histoire du pays, un rapport particulier du propriétaire au bien qu'il possède. La colonisation est un système qui se base sur la dépossession violente de l'autre de tout ce qu'il possède : mode de vie, biens matériels, femmes, enfants, terres... Une fois l'indépendance acquise, il est difficile d'effacer des esprits le profond traumatisme que peuvent causer 132 ans de violence et de déni. Le rapport au bien et à la propriété est alors affecté et nous pouvons en apercevoir les particularités dans le cas que nous offre Sansal dans son premier roman. Dans le cas algérien, la colonisation a dépossédé le peuple de ses terres et de sa dignité. Une fois installé, le système colonial a construit des villes et des domaines agricoles pour les colons. Ces villes n'étaient donc pas là avant le système colonial; elles ont été imaginées et conçues par le colon et pour le colon. L'indépendance est donc dans un sens une dépossession du colon de ce qu'il a bâti; l'Algérien, quant à lui, quarante années après l'indépendance acquise, habite le domaine du colon. Le roman de Sansal met en avant le fait que l'Algérien une fois l'indépendance recouvrée s'est rué sur «les domaines des colons» qui ne lui appartiennent pas afin non pas de «reposséder » un bien qui lui a été enlevé, mais de posséder un bien qui ne lui a jamais appartenu. Sansal, en excluant ce type "repossession», semble avaliser une vision positive du colonialisme: les domaines coloniaux seraient essentiellement le fruit d'un labeur du colon. Cette vision nourrit en effet la représentation du colon pionnier que les piedsnoirs ont largement reconduit dans leur représentation de l'Algérie française : transformation de marécages en terres agricoles fécondes non seulement en excluant la mention des processus de dépossession des indigènes des terres arables, mais aussi en omettant le rôle de la main d'œuvre (un sous-prolétariat corvéable à merci), pourtant indispensable, à l'enrichissement des propriétaires. 
Ce rapport complexe qu'entretient le sujet avec ce qu'il possède est pris en considération dans Dispossession: The performative in the political lorsque Butler rappelle qu'il faut faire attention aux différentes existences sociales que peut prendre le concept de propriété. Comme nous venons de le voir, l'histoire de l'Algérie a façonné de nouveaux rapports entre le propriétaire et ce qu'il possède. Butler et Athanasiou expliquent que : "We cannot understand what happens to an individual's land if we do not undestand both the social form of property and the social form of individuality. » (12)

Ce passage nous rappelle en effet que le concept de propriété peut prendre différentes formes selon le contexte social et historique. Dans le cas de l'Algérien, le concept de propriété est altéré et l'Algérien, quarante ans après l'indépendance, ne se sent pas pleinement propriétaire de l'endroit où il habite. Sansal, en appelant les fermes qui entourent la ville de Rouiba les "domaines des colons", rappelle que l'industrialisation et le terrorisme sont venus s'additionner aux profonds traumatismes qu'ont laissés 130 années de colonisation. Si l'on rajoute ce sentiment que ce qu'on possède n'est pas vraiment à soi, la dépossession que vit le citoyen de la ville de Rouiba est tridimensionnelle.

Enfin, un quatrième facteur de dépossession agit comme violence supplémentaire sur la société de la ville de Rouiba et, par extension, sur les Algériens. Dans un passage qui décrit le processus d'industrialisation de la ville, l'auteur fait référence à un évènement vécu par de nombreux propriétaires terriens algériens comme un véritable déchirement étant donné son ampleur. Les nationalisations qu'a connues l'Algérie pendant les vingt premières années de son indépendance sont un évènement important étant donnée leur portée culturelle et sociale. La Révolution agraire menée pendant les années soixante et soixante-dix avait pour principe la récupération par l'État de toutes les terres non travaillées. De nombreux litiges ont ainsi été enregistrés du fait que de nombreuses terres sont passées sous contrôle direct de l'État. Cet évènement est repris dans la citation suivante où Sansal décrit la transformation de la ville de Rouiba : «La mue ne s'est pas faite en un jour [...] Elle fut longue et douloureuse. $\mathrm{Au}$ commencement était l'ivresse. Puis vint le désenchantement. On implora le ciel, oubliant qu'il avait été nationalisé avec la terre et livré aux orties. On se mordit les doigts, mais la misère est insatiable. » (Sansal 9)

Dans cette citation, Sansal critique magistralement la Révolution agraire qui fut un échec pour le pays. L'auteur parle en effet de la nationalisation du ciel qui avait été « livré aux orties » avec la terre. L'ortie est un type de plantes qui poussent de façon sauvage et l'auteur fait référence ici à l'échec de la Révolution agraire qui a fait nationaliser de nombreuses terres pour ensuite les laisser à l'abandon. La Révolution agraire dont le but était de redistribuer les terres non travaillées aux paysans qui n'en avaient pas après les avoir nationalisées a généré de nombreux 
problèmes parmi lesquels l'omniprésence de l'État qui, finalement, était le seul à posséder ces terres sans que le paysan ne s'en sente propriétaire. Cette problématique est développée par Butler en citant le cas israélopalestinien. Butler explique en effet comment le concept de propriété a été remodelé par l'idéologie nationaliste israélienne afin de prendre possession des terres palestiniennes: "We can see how, in fact, the aims of both the nation and the colony depended upon an ideology of possessive individualism that was recast as possessive nationalism.» $(12,13)$. Butler parle dans cette citation de "nationalisme possessif», un nationalisme possessif qui est à l'œuvre dans le cas de la Révolution agraire algérienne, car en accaparant des terres cultivables, l'État se transforme en facteur de dépossession. La nationalisation implique, dans ce cas, la récupération par l’État de terres parfois déjà revendiquées.

\section{Conclusion}

Nous avons dans cette étude comment le terrorisme est représenté dans l'univers fictif du premier roman de Boualem Sansal Le serment des barbares et ce, en nous appuyant sur le concept de «dispossession» tel que théorisé par Butler et Athanasiou. Dans le cas de l'Algérie de la fin des années quatre-vingt-dix, l'auteur décrit des citoyens soumis à plusieurs violences qui agissent simultanément. Nous avons ainsi étudié comment se traduisent dans le texte de Sansal les effets de l'exposition prolongée au terrorisme qui crée chez l'individu un assujettissement volontaire qui se caractérise par l'abandon de l'individualité au profit du groupe. Nous avons aussi vu, dans le premier roman de Boualem Sansal, comment le terrorisme agit sur une société en proie à de multiples problèmes politiques et économiques. Nous avons relevé la dépossession du mode de vie et de la propriété que crée l'industrialisation qui agit sur la société comme une politique oppressante. Nous avons finalement vu comment ces deux facteurs de dépossession viennent se rajouter à d'autres couches de dépossessions qui se sont succédé à travers l'histoire du pays. Le terrorisme et l'industrialisation viennent en effet se rajouter au traumatisme encore vif de la colonisation dont nous avons analysé les effets sur le concept de la propriété dans le roman, mais aussi, à la nationalisation des terres lors de la Révolution agraire menée après l'indépendance du pays. 


\section{Bibliographie}

Athanasiou, Athena. Butler, Judith. Dispossession: The performative in the political, Polity Press, 2013.

Duday, H. Courtaud, P. Crubezy, E. Sellier, P. Tillier, A-M, «L'Anthropologie « de terrain»: reconnaissance et interprétation des gestes funéraires. » Bulletins et Mémoires de la Société d'anthropologie de Paris, n³. pp. 29-49, 1990, http://www.persee.fr/doc/bmsap 0037-

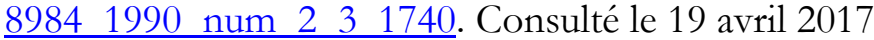

Goffman, Erving. Les rites d'interaction. Éditions de Minuit, 1974.

Sansal, Boualem. Le serment des barbares. Gallimard, 1999. 